\title{
A Class of Predictive Estimators in Two-Stage Design when Auxiliary Character is Estimated
}

\author{
Monika Saini* \\ School of Mathematics \& Computer Applications \\ Thapar University, Patiala, India-147001; mnksaini4@gmail.com
}

\begin{abstract}
This paper presents a class of predictive estimators for two stage sampling with unequal first and second stage units to the case where information on auxiliary character is not available. A double sampling procedure is proposed as alternative under such a situation. The proposed class consists of mainly two estimators namely Ratio and Regression. The mean square error (MSE) and minimum mean square of this class have been derived. In addition, we support these theoretical results by an empirical study.
\end{abstract}

Keywords: Double Sampling, Finite Population, Predictive Estimators, Two-stage Sampling.

\section{Introduction}

Auxiliary information has always been effective in increasing the precision of estimates in survey sampling. It is common practice to make use of auxiliary information on a auxiliary character $\mathrm{x}$ for the estimation of the finite population mean or total of a character under study. Laplace [4] was the first to use the auxiliary information in ratio type estimator. Watson [7] used regression method of estimation to estimate the average area of the leaves on a plant. The auxiliary information has been effectively used in two-phase sampling to estimate a population characteristic. A variety of approaches are available to construct more efficient estimators for the population mean and total, including design based and model based methods. In a predictive approach a model is specified for the population values and is used to predict the non sampled values. Prediction theory for sampling surveys (or model- based theory) can be considered as a general framework for statistical inferences on the character of finite population. Well-known estimators of population totals encounted in the classical theory, as expansion, ratio, regression, another estimators, can be predictors in a general prediction theory, under some special model. Srivastava [6] suggested the predictive estimator for product estimator also utilized the prediction criterion given by Basu [1]. Scott and Smith [5] described the predictive for two stage sampling. More recently Hossian and Ahmed [3] suggested some predictive estimators using an auxiliary variable.

In this paper, we propose a class of predictive estimators for two stage design when auxiliary character is estimate to construct respectively ratio and regression estimators by utilizing the prediction criterion.

* Corresponding author:

Monika Sainl (mnksaini4@gmail.com) 


\section{Suggested Procedure and Notations}

Consider a finite population $\mathrm{U}$ be partitioned into $\mathrm{N}$ First Stage Units (fsu) denoted by $\left(U_{\downarrow} 1, \ldots U_{\downarrow} i, \ldots U_{\downarrow} N\right)$ such that the number of Second Stage Units (ssu) in $U_{i}$ is $M_{i}$ and $M=\sum_{i}^{N} M_{i}$. When information on auxiliary character is not available, a double sampling procedure is proposed as an alternative under such a situation for the estimation of population mean $\bar{Y}$. Assume that a sample $s^{\prime}$ of $n^{\prime}$ denote the size of first phase sample of fsu (usually large) is drawn from $U$ by using SRSWOR for measuring information on auxiliary character $x$, let $\left\{x_{i}\right\}$ $\left(\mathrm{i}=1,2, \ldots, n^{\prime}\right)$ denote the $x$ observations and $\bar{x}^{\prime}=\frac{1}{n^{\prime}} \sum_{i}^{n^{\prime}} x_{i}$ the sample means. A second phase sample s of size $n\left(<n^{\prime}\right)$ is drawn as a subsample from $n^{\prime}$ i.e. the first-phase sample by using SRSWOR and then a sample $s_{i}$ of $m_{i}$ ssu's from the $i^{\text {th }}$ selected fsu $U_{i}$ of $M_{i}$ ssus according to the sampling scheme. We define symbolically

$$
\begin{array}{ll}
\bar{y}_{i}=\frac{1}{m_{i}} \sum_{j \in S_{i}} y_{i j} & \bar{y}=\frac{1}{n} \sum_{i \in S} w_{i} \bar{y}_{i} \\
\bar{x}=\frac{1}{n} \sum_{i \in S} w_{i} \bar{x}_{i} & \bar{y}^{\prime}=\frac{1}{n^{\prime}} \sum_{i \in S^{\prime}} w_{i} \bar{Y}_{i}
\end{array}
$$

and $\quad \bar{x}^{\prime}=\frac{1}{n^{\prime}} \sum_{i \in s^{\prime}} w_{i} \bar{Y}_{i}$

where $w_{i}=\frac{M_{i}}{\bar{M}}=\frac{N M_{i}}{M}$ also $\mathrm{M}=\sum_{i}^{N} M_{i}$ and $\bar{M}=\frac{1}{N} \sum_{i}^{N} M_{i}$

\section{Prediction Criterions and Proposed Class of Estimators}

When information on auxiliary character for $U$ is unknown and is collected by using double sampling, then the classical two-stage ratio and regression estimators of $\bar{Y}$ and their respective first order approximate mean square errors (MSE) are given as follows:
Consider the Ratio estimator

$$
\left(d_{1}\right)=\frac{\bar{y}}{\bar{x}} \bar{x}
$$

Then

$$
\begin{aligned}
\operatorname{MSE}\left(d_{1}\right)= & \left(\frac{1-f^{\prime}}{n^{\prime}}\right) S_{y}^{2}+\left(\frac{1-f}{n}\right)\left\{S_{y}^{2}-2 R S_{y x}+R^{2} S_{x}^{2}\right\} \\
& +\frac{1}{n N} \sum_{i=1}^{N} w_{i}^{2}\left(\frac{1-f_{i}}{m_{i}}\right)\left\{S_{i y}^{2}-2 R S_{i y x}+R^{2} S_{i x}^{2}\right\}
\end{aligned}
$$

Consider the Regression estimator

$$
\begin{aligned}
\left(d_{\downarrow} 2\right) & =y^{-}+b\left(x^{-\uparrow}-x^{-}\right) \\
& \approx \bar{y}+B\left(\bar{x}^{\prime}-\bar{x}\right)
\end{aligned}
$$

Then

$$
\begin{aligned}
\operatorname{MSE}\left(d_{2}\right)= & \left(\frac{1-f^{\prime}}{n^{\prime}}\right) S_{y}^{2}+\left(\frac{1-f}{n}\right)\left\{S_{y}^{2}-2 B S_{y x}+B^{2} S_{x}^{2}\right\} \\
& +\frac{1}{n N} \sum_{i=1}^{N} w_{i}^{2}\left(\frac{1-f_{i}}{m_{i}}\right)\left\{S_{i y}^{2}-2 B S_{i y x}+B^{2} S_{i x}^{2}\right\}
\end{aligned}
$$

where $R=\frac{Y}{\bar{X}}, B=\frac{S_{y x}}{S_{x}^{2}} f^{\prime}=\frac{n^{\prime}}{N}, f=\frac{n}{n^{\prime}} f_{i}=\frac{m_{i}}{M_{i}}$

$$
\begin{aligned}
& S_{y x}=\frac{1}{N-1} \sum_{i}^{N} \mathbb{Z}\left(w_{i} \bar{Y}_{i} \bar{I}-\bar{Y}\right)\left(w_{i} \bar{X}_{i}-\bar{X}\right) \\
& S_{i y}^{2}=\frac{1}{M_{i}-1} \sum_{j}^{M_{i}} \mathbb{Z}\left(y_{\tau_{i j}}-\bar{Y}_{i}\right)^{2}
\end{aligned}
$$

and $S_{y}^{z}$ and $S_{x}^{z}$ can be obtained from $S_{y x}$ using $\mathrm{y}=\mathrm{x}$ Under the usual predictive set-up, it possible to express, for a given non empty set s, we can partition

$$
\begin{aligned}
\bar{Y} & =\frac{\sum_{i}^{N} \sum_{j}^{M_{i}} y_{i j}}{\sum_{i}^{N} M_{i}}=\frac{\sum_{i}^{N} M_{i} \overline{Y_{i}}}{\sum_{i}^{N} M_{i}}=\frac{1}{M}\left[\sum_{i}^{N} M_{i} \overline{Y_{i}}\right] \\
& =\frac{1}{M}\left[\sum_{i \varepsilon s} M_{i} \overline{Y_{i}}+\sum_{i \varepsilon s^{\prime}} M_{i} \overline{Y_{i}}\right] \\
& =\frac{1}{M}\left[\sum_{i \varepsilon s}\left\{\sum_{i \varepsilon s} M_{i} \overline{Y_{i}}+\sum_{i \varepsilon \bar{s}} M_{i} \overline{Y_{i}}\right\}+\sum_{i \varepsilon \bar{s}^{\prime}} M_{i} \overline{Y_{i}}\right]
\end{aligned}
$$




$$
\begin{aligned}
= & \frac{1}{\mathrm{M}}\left[\sum _ { \mathrm { i } \varepsilon s ^ { \prime } } \left\{\sum_{\mathrm{i} \varepsilon s}\left(\sum_{\mathrm{j} \varepsilon s_{\mathrm{i}}} \mathrm{y}_{\mathrm{ij}}+\sum_{\mathrm{j} \varepsilon \bar{s}_{\mathrm{i}}} \mathrm{y}_{\mathrm{ij}}\right)\right.\right. \\
& \left.\left.+\sum_{\mathrm{i} \varepsilon \bar{s} \overline{\mathrm{s}}} \mathrm{M}_{\mathrm{i}} \overline{\mathrm{Y}_{\mathrm{i}}}\right\}+\sum_{\mathrm{i} \varepsilon \bar{s}^{\prime}} \mathrm{M}_{\mathrm{i}} \overline{\mathrm{Y}_{\mathrm{i}}}\right]
\end{aligned}
$$

where $\bar{s}^{\prime}$, denotes the set of $\left(N-n^{\mathrm{T}}\right)$ first phase of fsu's in U which are not included in $s^{\prime}, \bar{s}$ denoted the set of $\left(n^{\prime}-n\right)$ second phase of fsu's in $U$ which are not included in $\mathrm{s}$ and $\bar{s}_{i}$ the set of $\left(M_{\downarrow} i-m_{\downarrow} i\right)$ ssus of $U_{i}$ which are not included in $s_{i}, i=1,2, \ldots, n$.

$$
\begin{aligned}
\bar{Y}= & \frac{1}{M}\left[\sum_{i \varepsilon s^{\prime}}\left\{\sum_{i \varepsilon s}\left(m_{i} \overline{y_{i}}+\left(M_{i}-m_{i}\right) \overline{Y_{i r}}\right)\right\}\right] \\
& +\frac{1}{M} \sum_{i \varepsilon \bar{s}} M_{i} \overline{Y_{i}}+\frac{1}{M} \sum_{i \varepsilon \bar{s}^{\prime}} M_{i} \overline{Y_{i}}
\end{aligned}
$$

Let $\quad \bar{Y}_{r^{\prime}}=\frac{1}{N-n^{\prime}} \sum_{i \in \bar{S}^{\prime}} w_{i} \bar{Y}_{i}$

$$
\bar{Y}_{r}=\frac{1}{n^{\prime}-n} \sum_{i \in \bar{S}_{i}} w_{i} \bar{Y}_{i}
$$

and $\quad \bar{Y}_{i r}=\frac{1}{M_{i}-m_{i}} \sum_{j \in \bar{s}_{i}} y_{i j}$

We have

$$
\begin{aligned}
\bar{Y}= & \frac{1}{M}\left[\sum_{i \varepsilon s^{\prime}}\left\{\sum_{i \varepsilon s}\left(m_{i} \overline{y_{i}}+\left(M_{i}-m_{i}\right) \overline{Y_{i r}}\right)\right\}\right] \\
& +\frac{\left(n^{\prime}-n\right)}{N} \overline{Y_{r}}+\frac{\left(N-n^{\prime}\right)}{N} \overline{Y_{r}},
\end{aligned}
$$

where $\quad \bar{Y}_{r^{\prime}}=\frac{N \bar{Y}-n^{\prime} \bar{y}^{\prime}}{N-n^{\prime}}$

$$
\bar{Y}_{r}=\frac{n^{\prime} \bar{y}^{\prime}-n \bar{y}}{n^{\prime}-n}
$$

and $\quad \bar{Y}_{i r}=\frac{M_{i} \bar{Y}_{i}-m_{i} \bar{y}_{i}}{M_{i}-m_{i}}$

To estimate $\bar{Y}$, we therefore have to predict the quantities $\bar{Y}_{i r}, \bar{Y}_{r}$ and $\bar{Y}_{r^{\prime}}$ from the sample data because the first component of the right hand side of (3.7) is already known. Using $Z_{r^{\prime}} Z_{r}$ and $Z_{i r}$ as their respective predictors,

Then, the predictive estimator of the population mean $\bar{Y}$ is

$$
\begin{aligned}
(\hat{\bar{Y}})_{\text {pre }}= & \frac{1}{M}\left[\sum_{i \varepsilon s^{\prime}}\left\{\sum_{i \varepsilon s}\left(m_{i} \overline{y_{i}}+\left(M_{i}-m_{i}\right) Z_{i r}\right)\right\}\right] \\
& +\frac{\left(n^{\prime}-n\right)}{N} Z_{r}+\frac{\left(N-n^{\prime}\right)}{N} Z_{r}^{\prime}
\end{aligned}
$$

where $Z_{r^{\prime}}, Z_{r}$ are the predictors of $\bar{Y}_{r^{\prime}}, \bar{Y}_{r}$ for first stage unit using double sampling and $Z_{i r}$ is the predictor of $\bar{Y}_{i_{r}}$ for second stage unit respectively.

In equation (3.8) we combine the last two terms i.e. Non sampled part of first phase and second phase in first stage units because in first stage using double sampling when we go from one phase to second sampling unit is not changed and non sampled units are $N-n^{\prime}+n^{\prime}-n=N-n$.

Equation (3.8) can be written as

$\left.(\hat{\bar{Y}})_{\text {pre }}=\frac{1}{M}\left[\sum_{\text {ies }} m_{i} \bar{y}_{i}+\left(M_{i}-m_{i}\right) Z_{i r}\right)\right]+\frac{(N-n)}{N} Z_{r}^{*}$

Define, $Z_{r}^{*}$ and $Z_{i r}$ as the class of estimators using single auxiliary variable $x$ for first stage unit using double sampling and second stage unit proposed as

$$
\begin{aligned}
& z_{r}^{*}=\bar{y}+t\left(\overline{x_{r}}-\bar{x}\right), \\
& z_{i r}=\overline{y_{i}}+t_{i}\left(\overline{x_{i r}}-\bar{x}_{i}\right)
\end{aligned}
$$

and $t$ and $t_{i}$ are suitably chosen statistics for defining ratio and regression estimators (Table 1).

Now for first order approximation the MSE (3.10) are given respectively

$$
\begin{aligned}
& \operatorname{MSE}\left(Z_{r}^{*}\right)=\left(\frac{1-f^{\prime}}{n^{\prime}}\right) S_{y}^{2}+\left(\frac{1-f}{n}\right) \\
&\left\{S_{y}^{2}-2 T \rho S_{x} S_{y}+S_{y}^{2} S_{x}^{2}\right\} \\
& \operatorname{MSE}\left(Z_{i r}\right)=\left(\frac{1-f_{i}}{m_{i}}\right)\left\{S_{i y}^{2}-2 T i \rho S_{i x} S_{i y}+S_{i y}^{2} S_{i x}^{2}\right\}
\end{aligned}
$$

where equation (3.11) is the mean square error for first stage unit using double sampling and equation (3.12) is the mean square error for second stage unit respectively.

If we put different value of $T$ and $T_{i}$ are given below then we get the various types of estimators.

Table 1

\begin{tabular}{lcccc}
\hline Estimator & \multicolumn{4}{c}{ Different values } \\
\hline \multirow{2}{*}{ Ratio } & $t$ & $t_{i}$ & $T$ & $T_{i}$ \\
& $\bar{y} / I_{\bar{X}}$ & $\bar{y}_{i} / \bar{x}_{i}$ & $\bar{Y} / \bar{X}$ & $\bar{Y}_{i} / \bar{X}_{i}$ \\
Regression & $\mathrm{b}$ & $b_{i}$ & $\mathrm{~B}$ & $B i$ \\
\hline
\end{tabular}


Ratio Estimator:

$$
\begin{aligned}
& \operatorname{MSE}\left(Z_{r}^{*}\right)=\left(\frac{1-f^{\prime}}{n^{\prime}}\right) S_{y}^{2}+\left(\frac{1-f}{n}\right)\left\{S_{y}^{2}-2 R \rho S_{x} S_{y}+R^{2} S_{x}^{2}\right\} \\
& \operatorname{MSE}\left(Z_{i r}\right)=\left(\frac{1-f_{i}}{m_{i}}\right)\left\{S_{i y}^{2}-2 R i \rho S_{i x} S_{i y}+R_{i}^{2} S_{i x}^{2}\right\}
\end{aligned}
$$

Regression Estimator:

$\operatorname{MSE}\left(Z_{r}^{*}\right)=\left(\frac{1-f^{\prime}}{n^{\prime}}\right) S_{y}^{2}+\left(\frac{1-f}{n}\right)\left\{S_{y}^{2}-2 B \rho S_{x} S_{y}+B^{2} S_{x}^{2}\right\}$

$$
\operatorname{MSE}\left(Z_{\text {ir }}\right)=\left(\frac{1-f_{i}}{m_{i}}\right)\left\{S_{i y}^{2}-2 B i \rho S_{i x} S_{i y}+B_{i}{ }^{2} S_{i x}^{2}\right\}
$$

Then the proposed class of predictive estimators is

$(\hat{\bar{Y}})_{\text {pre }}=\frac{1}{M}\left[\sum_{i \varepsilon s^{\prime}} \sum_{i \varepsilon s}\left(M_{i}\left(\overline{y_{i}}+t_{i}\left(\overline{X_{i}}-\bar{X}_{i}\right)\right)\right]+\left(\bar{y}+t\left(\overline{X^{\prime}}-\bar{x}\right)\right)\right.$

Assume that $E(t)=E_{1} E_{2}(t)=T$ or $E(t) \approx T$ and $E\left(t_{i}\right)=T_{i}$ or $E\left(t_{i}\right) \approx T_{i}$. The choices of $t, t_{i}, T$ and $T_{i}$ for different predictive estimators are presented in the following Table 1

From Table 1 after simple algebraic manipulations $(\hat{\bar{Y}})_{\downarrow}$ pre turns out to be ratio and regression estimators respectively:

$$
\begin{aligned}
& d_{01}=d_{1}+\frac{1}{N} \sum_{i} w_{i}\left(\frac{\overline{y_{i}}}{\overline{x_{i}}}-\frac{\bar{y}}{\bar{x}}\right) \overline{x^{\prime}} \\
& d_{02}=d_{2}+\frac{1}{N} \sum_{i} w_{i}\left[\left(\overline{y_{i}}+b_{i}\left(\overline{X_{i}}-\overline{x_{i}}\right)+(\bar{y}+b(\bar{X}-\bar{x}))\right]\right.
\end{aligned}
$$

\section{The Efficiency of Estimators}

After some simplification, we have the first order of mean square error of $(\hat{\bar{Y}})_{\downarrow}$ pre $\left(d_{01}\right.$ and $\left.d_{\downarrow} 02\right)$ is as follows:

$$
\begin{aligned}
M\left(\hat{P}_{p r e}\right)= & \left(\frac{1-f^{\prime}}{n^{\prime}}\right) S_{y}^{2}+\left(\frac{1-f}{n}\right) \\
& \left\{S_{y}^{2}+T^{2} S_{x}^{2}-2 T S_{y x}\right\} \\
& +\frac{1}{n} \sum_{i}^{N} w_{i}^{2}\left(\frac{1-f_{i}}{m_{i}}\right) \\
& \left\{S_{i y}^{2}+\alpha_{i}^{2} S_{i x}^{2}-2 \alpha_{i} S_{i y x}\right\}
\end{aligned}
$$

where $\alpha_{i}=T-f\left(T-T_{i}\right)$
The optimum values of $\mathrm{T}$ and $T_{i}$ which minimize $M\left(\hat{P}_{\text {pre }}\right)$ are given respectively as

$$
\begin{aligned}
& T_{\text {opt }}=\frac{S_{y x}}{S_{x}^{z}}=B_{b} \\
& T_{\text {iopt }}=\frac{S_{i y x}}{S_{i x}^{z}}=B_{i b}
\end{aligned}
$$

Then the minimum mean square error of $\widehat{\bar{Y}}$ is

$$
\begin{aligned}
M(\hat{Y})_{o p t}= & \left(\frac{1-f^{\prime}}{n^{\prime}}\right) S_{y}^{2}+\left(\frac{1-f}{n}\right) \\
& \left\{S_{y}^{2}+T_{o p t}^{2} S_{x}^{2}-2 T_{o p t} S_{y x}\right\} \\
+ & \frac{1}{n} \sum_{i}^{N} w_{i}^{2}\left(\frac{1-f_{i}}{m_{i}}\right) \\
& \left\{S_{i y}^{2}+\alpha_{i o p t}^{2} S_{i x}^{2}-2 \alpha_{i o p t} S_{i y x}\right\} \\
M(\hat{Y})_{o p t}= & \left(\frac{1-f^{\prime}}{n^{\prime}}\right) S_{y}^{2}+\left(\frac{1-f}{n}\right) \\
& \left\{S_{y}^{2}+B_{b}^{2} S_{x}^{2}-2 B_{b} S_{y x}\right\} \\
& +\frac{1}{n} \sum_{i}^{N} w_{i}^{2}\left(\frac{1-f_{i}}{m_{i}}\right) \\
& \left\{S_{i y}^{2}+A_{\alpha}^{2} S_{i x}^{2}-2 A_{\alpha} S_{i y x}\right\}
\end{aligned}
$$

where $\alpha_{\text {iopt }}=A_{\alpha}$

where is the mean square error of regression estimator. Hence regression estimator is the optimum estimator of this class.

The minimum mean square error of $d_{01}$ and $d_{02}$ up to the first order approximation are given respectively as follows:

Ratio Estimator:

$$
\begin{aligned}
M\left(d_{o 1}\right)= & \left(\frac{1-f^{\prime}}{n^{\prime}}\right) S_{y}^{2}+\left(\frac{1-f}{n}\right) \\
& \left\{S_{y}^{2}+R^{2} S_{x}^{2}-2 R S_{y x}\right\} \\
& +\frac{1}{n} \sum_{i}^{N} w_{i}^{2}\left(\frac{1-f_{i}}{m_{i}}\right) \\
& \left\{S_{i y}^{2}+\alpha_{i}^{2} S_{i x}^{2}-2 \alpha_{i} S_{i y x}\right\}
\end{aligned}
$$


Regression Estimator:

$$
\begin{aligned}
M\left(d_{o z}\right)= & \left(\frac{1-f^{\prime}}{n^{\prime}}\right) S_{y}^{2}+\left(\frac{1-f}{n}\right) \\
& \left\{S_{y}^{2}+B^{2} S_{x}^{2}-2 B S_{y x}\right\} \\
& +\frac{1}{n} \sum_{i}^{N} w_{i}^{2}\left(\frac{1-f_{i}}{m_{i}}\right) \\
& \left\{S_{i y}^{2}+\gamma_{i}^{2} S_{i x}^{2}-2 \gamma_{i} S_{i y x}\right\}
\end{aligned}
$$

where $\alpha_{i}=R-f\left(R-R_{i}\right)$ and $\gamma_{i}=B-f\left(B-B_{i}\right)$

Subtracting the equation (4.2) and (4.3) from the equation (3.2) and (3.4) respectively, we get

Difference: MSE $\left(d_{j}\right)-M S E\left(d_{0 j}\right)=D_{j}$ where $j=1,2$

$$
\begin{aligned}
& D_{1}=\frac{1}{n N} \sum_{i}^{N} w_{i}^{2}\left(\frac{1-f_{i}}{m_{i}}\right)\left\{\left(R^{2}-\alpha_{i}^{2}\right) S_{i x}^{2}-2\left(R-\alpha_{i}\right) S_{i y x}\right\} \\
& D_{1}=\frac{1}{n N} \sum_{i}^{N} w_{i}^{2}\left(\frac{1-f_{i}}{m_{i}}\right)\left\{\left(B^{2}-\gamma_{i}^{2}\right) S_{i x}^{2}-2\left(B-\gamma_{i}\right) S_{i y x}\right\}
\end{aligned}
$$

Thus $d_{01}$ and $d_{02}$ will be more efficient than $d_{1}$ and $d_{2}$ respectively

$$
\text { If } \begin{aligned}
\beta_{i y x} & \leq \frac{R+\alpha_{i}}{2} \quad \mathrm{i}=1,2, \ldots, \mathrm{N} \text { for ratio } \\
\delta_{i y x} \leq \frac{B+\gamma_{i}}{2} & \text { for regression }
\end{aligned}
$$

where $\beta_{i y x}=\frac{S_{i y x}}{S_{i x}^{2}}$ and $\delta_{i y x}=\frac{S_{i y x}}{S_{i x}^{2}}$ be the regression coefficient of $\mathrm{y}$ on $\mathrm{x}$ in $U_{i}$.

\section{Empirical Study}

To show the usefulness of suggested methodology presented in this paper, numerically, an empirical study has been carried out.

Population: We consider the 2001 census data which relates to the total number of agricultural laboures and the total no. of cultivators of 444 villages of Bhiwani district of Haryana (Table 2). We take no. of agricultural laboures in villages as $\mathrm{y}$ and the total no. of cultivators in villages as $\mathrm{x}$. The whole population of Bhiwani district (444 villages) is divided into 9 blocks (fsus) where $i^{\text {th }}(\mathrm{i}=1,2, \ldots, 9)$ block consists of $M_{i}$
Table 2. Description of Population

\begin{tabular}{lc}
\hline Source & $\begin{array}{c}\text { Village wise information of Bhiwani District } \\
\text { of Haryana (2001 census data) }\end{array}$ \\
\hline$y$ & study variable ( agricultural labourers) \\
$x$ & auxiliary information (total no. of cultivators) \\
\hline
\end{tabular}

Table 3. Percentage relative efficiencies of proposed estimators
(i)
(ii)
(iii)
(iv)

\begin{tabular}{lccc} 
Estimators & $\begin{array}{c}\text { Auxiliary } \\
\text { variable used }\end{array}$ & Variance $\times \mathbf{1 0}^{\mathbf{7}}$ & R.E \% w.r.t. $\boldsymbol{d}_{1}$ \\
\hline$d_{1}$ & $x$ & 2.55 & 100 \\
$d_{01}$ & $x$ & 2.45 & 104 \\
& $x$ & 2.62 & R.E \% w.r.t. $\boldsymbol{d}_{2}$ \\
$d_{2}$ & $x$ & 2.49 & 100 \\
$d_{02}$ & $x$ & 105 \\
\hline
\end{tabular}

villages (ssus), $i=1,2,3, \ldots . ., 9, M_{1}=25, M_{2}=56, M_{3}=32$, $M_{4}=45, M_{5}=36, M_{6}=78, M_{7}=66, M_{8}=55 M_{9}=51$ ). The numerical values of the estimate of population mean, its variance were worked out under each of the proposed estimators from population values. Subsequently, the relative efficiency of the estimators given in this paper was calculated. Variance and percent relative efficiency of different estimators using auxiliary information in two stage design are obtained in Table 3.

\section{Conclusion}

Section 4 provides the conditions under which the estimators $d_{01}$ and $d_{02}$ have less mean squared error as compared to $d_{1}$ and $d_{2}$ when auxiliary character is estimated in two stage design.

Table 3 shows that the estimators $d_{01}$ and $d_{02}$ has highest percent relative efficiency w.r.t. to $d_{1}$ and $d_{2}$. Thus the estimator $d_{01}$ and $d_{02}$ are recommended for used in practice for the estimation of population mean.

\section{References}

1. Basu D (1971). An essay on the logical foundations of survey sampling, Part-I: In Foundations of statistical Inference, Ed. By Godambe V P and Spott D A, Toronto: Holt, Rinehart \& Winston, 203-237.

2. Chaudhari A, and Stenger H (1992). Survey Sampling: Theory and Methods, Maracel Dekker, Inc., New York.

3. Hossian M I, and Ahmed, M S (2001). A class of Predictive estimators in Two-Stage sampling using Auxiliary infor- 
mation, Information and management Sciences, vol 12(1), 49-55.

4. Laplace P S (1820). A philosophical essay on probabilities, English Translation, Dover, 1951.

5. Scott A, and Smith T M P (1969). Estimation in Multistage surveys, Journal of the American Statistical Association, 64(327), 830-840.
6. Srivastava S K (1983). Predictive Estimation of finite population mean using product estimator, Metrika, 30(1), 93-99.

7. Watson D J (1937). The estimation of leaf areas in field crops, Journal of Agricultural Science,, 27(3), 474-483. 